\title{
How to study G-quadruplex structures
}

\author{
Magdalena Matgowska *, Dorota Gudanis *, Anna Teubert, Grażyna Dominiak, Zofia Gdaniec ** \\ Institute of Bioorganic Chemistry, Polish Academy of Sciences, Poznań, Poland \\ **Corresponding author: zgdan@ibch.poznan.pl \\ ( ${ }^{*}$ These two authors have contributed equally)
}

\begin{abstract}
All guanine-rich DNA and RNA molecules tend to fold into inter- and intra-molecular structures called G-quadruplexes. The core of a G-quadruplex is composed of at least two adjacent G-tetrads stabilized by monovalent cations. While it has been suggested that there could potentially be over 375000 quadruplex forming sequences in the human genome alone, only a small number of three-dimensional G-quadruplex structures are known. More structural data are needed to explain their presence and function in vivo. We offer here a short review of experimental methods commonly used to determine G-quadruplex topologies.
\end{abstract}

Key words: G-quadruplex, UV spectroscopy, CD spectroscopy, mass spectrometry, NMR spectroscopy

\section{Introduction}

In 1910 Bang (Bang, 1910) reported that concentrated solutions of guanosine easily self-associate and form polycrystalline gels. This unusual phenomenon puzzled researchers for the next five decades until 1962 (Gellert et al., 1962) when the association of guanine bases into a tetrameric arrangement was first determined by crystallographic methods, revealing the assembly of tetrameric units into large helical structures. For the next 25 years little attention was paid to the possible role of G-quadruplexes in biological systems until the observation that oligonucleotides corresponding to the G-rich strand of telomeric DNA displayed unexpectedly high electrophoretic mobility on nondenaturing polyacrylamide gels (Williamson et al., 1989). Two laboratories independently proposed that G-rich sequences in the chromosomes of eukaryotic cells, including the single-stranded 3 '-overhang of telomeres, could form four stranded DNA structures (Williamson et al., 1989; Sen et al., 1988).

Currently, there is considerable interest in quadruplexes because G-rich sequences with the potential to form this type of structure have been found to exist in several biologically important DNA regions, such as telomeres and the promoter regions of human oncogenes. Bioinformatic analysis has identified 375000 candidate sequences within the human genome that could adopt G-quadruplex structures, although many are likely to be due to chance and may be relatively unstable (Huppert et al., 2005; Todd et al., 2005). Another bioinformatic study has revealed that not only DNA but also numerous mRNA that contain G-rich sequences may be able to fold into G-quadruplex structures (Huppert et al., 2008a) suggesting that the RNA quadruplexes may be involved in biological processes.

G-quadruplex structures are important not only because they are involved in the regulation of transcription and translation (Di Antonio et al., 2012, Huppert, 2008b). There is increasing pharmaceutical interest in the potential possibility of manipulating these processes to develop novel therapeutics (Bugaut et al., 2012; Balasubramanian et al., 2009; Collie et al., 2011). Knowledge of G-quadruplex structures is essential for the rational design of small molecules that could selectively bind to these structures to specifically modulate gene transcription, or to inhibit telomerase, as well as for the development of aptamers that can selectively bind to particular proteins (Collie et al., 2011; Gatto et al., 2009). Recently, experimental evidence for the presence and function of G-quadruplexes in vivo has started to emerge (Lipps et al., 2009).

The number of potential applications of quadruplexes in supramolecular chemistry and material science is enormous, ranging from the formation of biosensors (Guo et al., 2012) and nanomachines (Alberti et al., 2006) to G-wires (Miyoshi et al., 2011) and they may serve as a basis for developing advanced biomaterials. 
The high potential of guanosine and its derivatives for self-recognition and self-assembly as well as their recognition ability for other biologically important molecules has been noted by the scientific community. The design of novel guanosine-based molecular architectures and their exploration as possible building blocks for new hybrid biomolecular electronics and biosensing was one of the objectives of the COST (European Cooperation in Science and Technology) Action MP0802 (2008-2012).

The fundamental unit of the quadruplex is a G-tetrad composed of a planar array of four guanine bases associated through a network of hydrogen bonds. Each guanine base is both a donor and acceptor of two hydrogen bonds, respectively (Fig. 1A). Quadruplex formation requires the presence of monovalent cations, in particular $\mathrm{K}^{+}$and, to a lesser extent, $\mathrm{Na}^{+}$or $\mathrm{NH}_{4}{ }^{+}$. The precise location of the cations between the tetrads depends on the nature of the ion. The $\mathrm{Na}^{+}$ions lie on a plane with a G-tetrad, whereas the $\mathrm{K}^{+}$and $\mathrm{NH}_{4}^{+}$ions exist between two successive G-tetrads. Quadruplexes can be formed by the association of four separate DNA or RNA strands, the dimerization of two strands that each contain two G-tracts, or the intramolecular folding of a single strand that contains four (or more) tracks of two or more guanine residues. Quadruplex structures can be parallel when all the strands are parallel or anti-parallel when at least one of four strands is anti-parallel to the others (Fig. 1B). In particular, a parallel quadruplex conformation implies that all nucleosides in individual G-tetrads have the same conformation of the glycosidic bond $($ anti $\bullet$ anti $\bullet$ anti $\bullet$ anti or $s y n \bullet$ syn $\bullet$ syn $\bullet$ syn). G-tetrads in anti-parallel quadruplexes comprise two guanosines in syn conformation and another two in anti conformation $(s y n \bullet$ syn $\bullet$ anti $\bullet$ anti and $s y n \bullet a n t i \bullet s y n \bullet a n t i)$. In the hybrid type, parallel-antiparallel (3+1) G-quadruplexes, three guanosines have the same conformation, while the fourth residue has an opposite one $($ syn $\bullet$ anti $\bullet$ anti $\bullet$ anti and anti $\bullet$ syn $\bullet$ syn $\bullet$ syn). The guanine tracts are connected by loops of variable size and sequence. There are three different types of loops (Fig. 2). Edgewise loops (also known as lateral loops) connect two adjacent antiparallel strands. Diagonal loops connect two opposing anti-parallel strands, while the double chain reversal loops (also known as propeller loops) connect the adjacent parallel strands. Both the length and the composition of the loops connecting the G-tetrads can influence the topology of a quadruplex and its thermodynamic sta- bility. All quadruplex structures have four grooves, the dimensions of which (narrow, medium and wide) are defined by the glycosidic conformation of the adjacent bases in the tetrad. For a more comprehensive description concerning the fundamentals of a quadruplex reference structure Burge et al. (2006) provides an excellent overview.

G-quadruplex structures reveal a high degree of structural polymorphism in terms of nucleotide sequence, the orientation of the strands, the syn/ anti glycosidic conformation of guanines, groove widths, the sequence and length of the loops or the nature of counter ions. Understanding the molecular forces that govern quadruplex folding is of particular interest. Many experimental techniques have been used to study G-quadruplex formation, each examining different aspects of the structure. Although the combined use of a few methods provides invaluable structural information on the conformational changes which occur under various conditions (concentration, $\mathrm{pH}$, temperature, salt type and its concentration, type of buffer, etc.). However, only X-ray crystallography and NMR spectroscopy allow complete structure determination with atomic resolution. Here, we describe the most common techniques which, in our opinion, are essential in G-quadruplex structural studies.

\section{Circular Dichroism Spectroscopy (CD)}

Circular dichroism spectroscopy (CD) is one of the simplest methods that can be used to assess the nature of the quadruplex fold. This technique relies on the differential absorption by chiral molecules of left and righthanded circularly polarized light (Boczkowska, 2002). $\mathrm{CD}$ spectra of nucleic acids are very sensitive to stacking interactions between the bases. In G-quadruplexes, three different types of topology (parallel, antiparallel, hybrid) are correlated with the syn/ anticonformation of guanosine involved in G-tetrad formation. As a result, different stacking is observed between adjacent G-tetrads and therefore a unique $\mathrm{CD}$ signature for a given topology occurs (Fig. 3) (Karsisiotis et al., 2011; Randazzo et al., 2012; Vorlickova et al., 2012). In general, CD spectra of typical four stranded parallel DNA quadruplexes exhibit absorption maxima at $264 \mathrm{~nm}$ and minima at $240 \mathrm{~nm}$. They can be easily distinguished from single or double stranded parallel quadruplexes with external loops, because an additional positive peak near $290 \mathrm{~nm}$ 
A

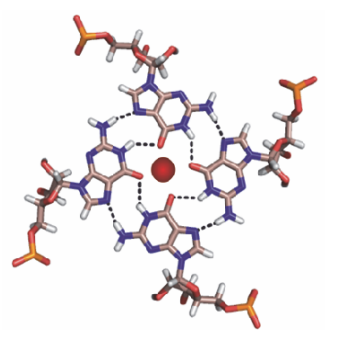

B

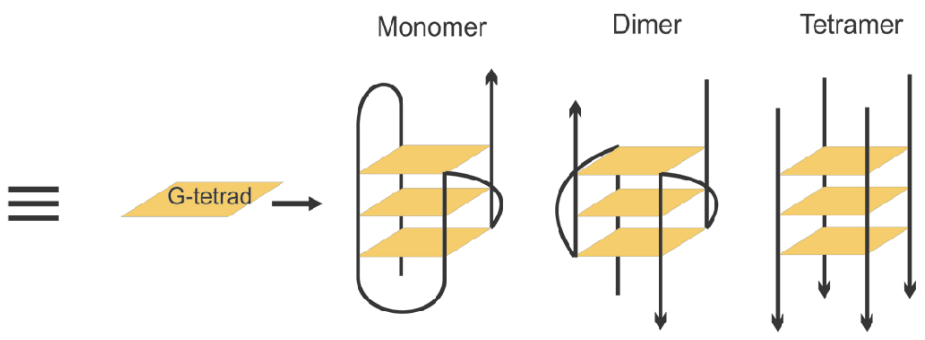

Fig. 1. Schematic representation of G-tetrad (A) and examples of possible quadruplex folds: anti-parallel single stranded, bimolecular anti-parallel and tetramolecular parallel

A

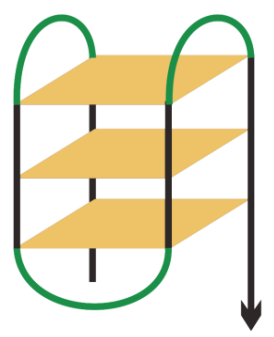

B

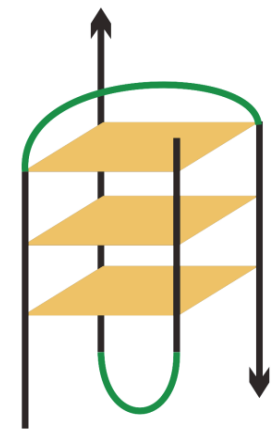

C

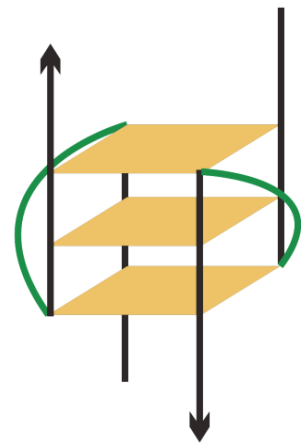

Fig. 2. Schematic diagrams showing potential linking loop arrangements: edgewise (A), diagonal (B) and double chain reversal loops (C)

A

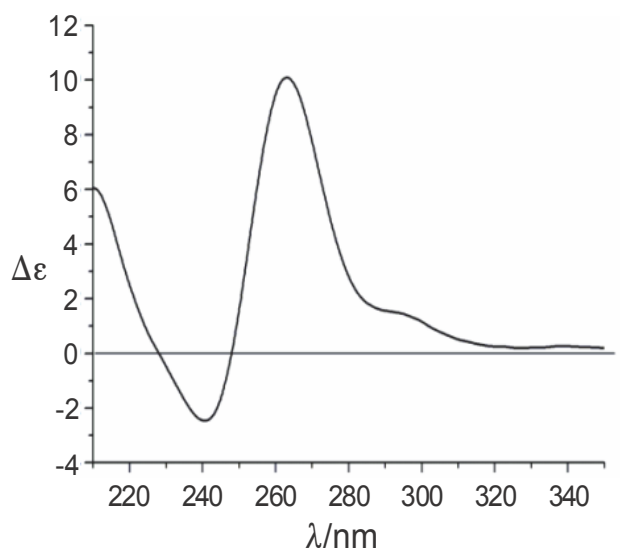

B

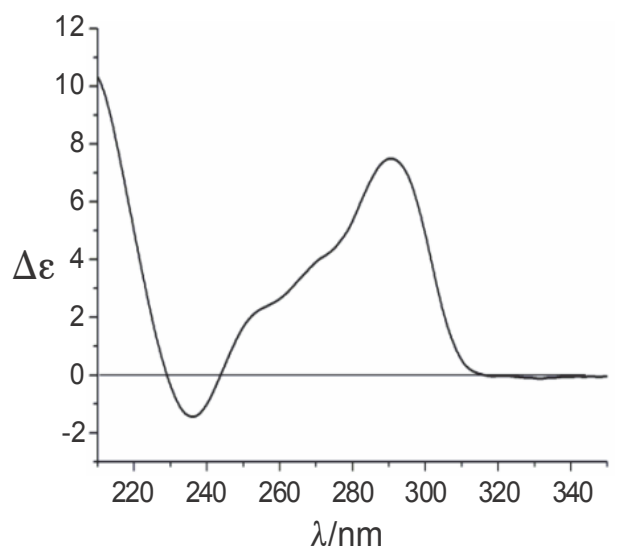

Fig. 3. The CD spectrum of parallel (A) and anti-parallel (B) DNA quadruplexes

appears in their CD spectra (Paramasivan et al., 2007). The CD spectra of DNA and RNA parallel quadruplexes are also very similar to those of A-form duplexes (Kypr et al., 2009). However, these two types of structural motifs can usually be distinguished by the positive or negative sign of a peak present at $210 \mathrm{~nm}$. A-form duplexes display a characteristic negative band, whereas parallel quadruplexes have a positive band at this wavelength. Thus, only the presence of two positive peaks, at $210 \mathrm{~nm}$ and 260 $\mathrm{nm}$, is indicative of the quadruplex structure. The appearance of an absorption maximum at $295 \mathrm{~nm}$ and minimum at $265 \mathrm{~nm}$ is characteristic of anti-parallel DNA quadru- 
Table 1. The characteristic peaks observed in CD spectra for different topologies of quadruplexes

\begin{tabular}{l|c|c|c|c}
\hline \multirow{2}{*}{\multicolumn{1}{c|}{ Type of the quadruplex }} & \multicolumn{4}{c}{ CD profile at $\lambda[\mathrm{nm}]$} \\
\cline { 2 - 5 } & 210 & 240 & 265 & 295 \\
\hline Tetramolecular parallel & + & - & + & \\
\hline $\begin{array}{l}\text { Bimolecular or unimolecular parallel } \\
\text { with external loops }\end{array}$ & + & - & + & + \\
\hline Antiparallel single stranded & + & + & - & + \\
\hline Hybrid (3+1) & + & - & + & + \\
\hline
\end{tabular}

plexes. Finally, the spectrum of hybrid quadruplexes is characterized not only by dominant positive bands at $295 \mathrm{~nm}$ and $265 \mathrm{~nm}$ but also a negative band at $240 \mathrm{~nm}$ (Table 1).

CD spectroscopy can also provide information on conformational changes induced by altered solution conditions. Using this technique one is able to follow the kinetics of quadruplex formation (Liu et al., 2012). It is also possible to analyze the map of conformational changes because the same molecule can isomerize due to many factors. For example, the addition of a molecular crowding agent such as poly(ethylene glycol) or co-solvent (methanol, ethanol, acetonitrile) induces structural changes in quadruplexes which can be observed by CD spectroscopy (Wei et al., 2009). Furthermore, analysis of the temperature dependence of the CD spectra allows the monitoring of the stability of qiuadruplex structure and a determination of the melting temperature. By minor modifications of the oligonucleotide sequence and careful optimization of solution conditions it is possible to obtain a single conformer in solution, which is crucial for the study of quadruplex structures by NMR spectroscopy and X-ray crystallography.

A better understanding of the various factors that contribute to the specific patterns of $\mathrm{CD}$ spectra is required before $\mathrm{CD}$ data alone can be used to determine quadruplex topology unambiguously (Gray et al., 2008). Until such data are available, the results obtained from CD spectra must be analyzed with caution and other biophysical methods should be used to confirm quadruplex formation.

\section{UV Spectroscopy}

\section{Profile of the melting curve}

UV spectroscopy is a fast method to confirm quadruplex structure formation and evaluate its structural sta- bility. Typically, measurements of the melting temperatures of nucleic acids are performed at a wavelength of $260 \mathrm{~nm}$, at which the maximum of their absorption is observed. For DNA and RNA duplexes approximately a $25 \%$ difference in absorption at $260 \mathrm{~nm}$ between the completely folded and unfolded (melted) states are detected. However, for DNA and RNA quadruplexes those differences are not that evident as they barely amount to $4 \%$. Optionally, it is possible to record the melting profile at different wavelengths, preferably at $295 \mathrm{~nm}$, where the absorbance is significantly lower, but denaturation of the sample is exhibited in an over 50\% change in absorbance amplitude (Mergny et al., 1998, Rachwal et al., 2007). A detailed protocol for UV melting studies of quadruplexes was prepared by J.L. Mergny and L. Lacroix (2009).

Confirmation of quadruplex structure formation is possible by simple analysis of the UV curve. This is because at $295 \mathrm{~nm}$ the melting profile of a quadruplex is inverted in relation to the profile of duplex structure. G-quadruplexes undergo a hypochromic shift (i.e. lower absorbance) at $295 \mathrm{~nm}$ upon melting (Fig. 4). Measurements of melting profiles in varying conditions enable a characterization of the dependence of the stability and kinetics of the quadruplex association and dissociation processes on various factors, including strand concentration, type and concentration of various ions. In addition, the thermodynamic parameters of this structure formation may be determined (Mergny et al., 1998 and 2005a; Gros et al., 2007; Smargiasso et al., 2008; Pasternak et al., 2011; Avino et al., 2012).

\section{Thermal Difference Spectra (TDS)}

A thermal difference spectrum (TDS) is obtained by subtracting the UV spectra recorded above and below the melting temperature (usually at $20^{\circ} \mathrm{C}$ and $90^{\circ} \mathrm{C}$, respectively). It has a shape that is unique for each type of 


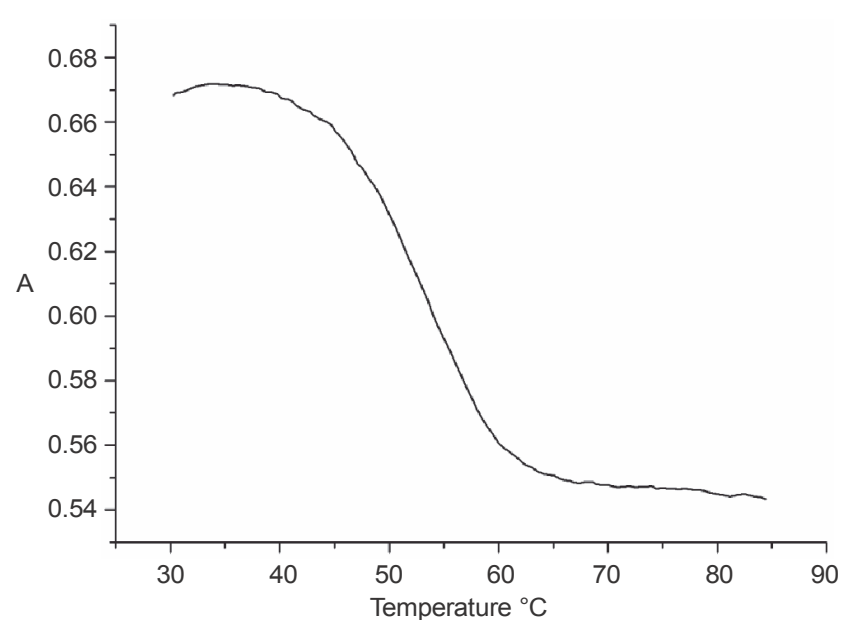

Fig. 4. UV denaturation profile of RNA quadruplex at $295 \mathrm{~nm}$

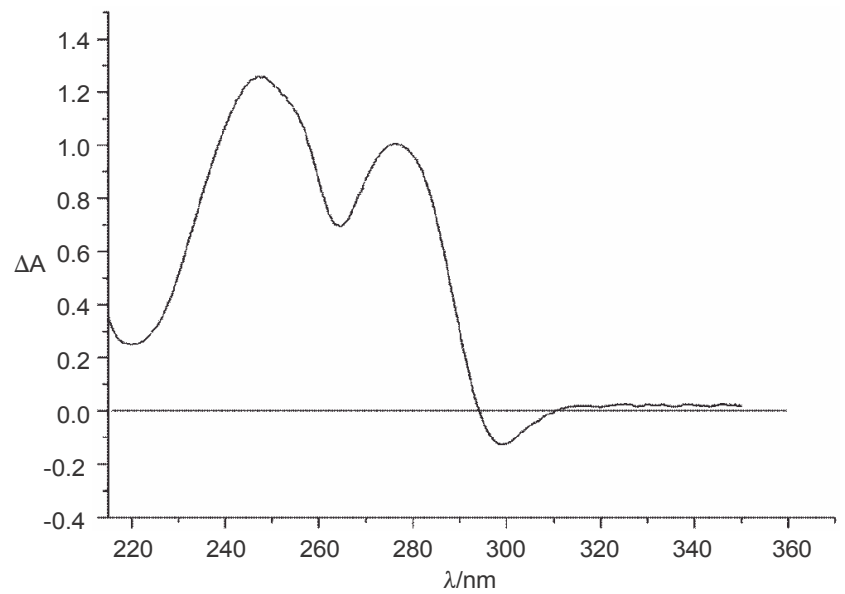

Fig. 5. An example of the thermal difference spectrum of RNA quadruplex

nucleic acid structure. A detailed description of the TDS profiles of nucleic acid structural motifs can be found in the literature (Mergny et al., 2005b). Over 900 TDS spectra were recorded for 200 different oligonucleotides that were representative for each type of possible folding. It is assumed that every structural motif is characterized by unique base stacking interactions, which is reflected in the TDS profile.

The TDS "fingerprint" of G-quadruplexes is characterized by a negative band at $295 \mathrm{~nm}$ and positive bands centered at about $240 \mathrm{~nm}$ and $270 \mathrm{~nm}$ (Fig. 5). The most characteristic is the band at $295 \mathrm{~nm}$, because it corresponds to the stacking interactions between G-tetrads that form the spine of the quadruplex structure. In the case of parallel quadruplexes the negative band is less intense than the band occurring for anti-parallel quadruplexes. A.T. Karsisiotis and colleagues (2011) proposed another TDS strategy to distinguish quadruplex topology. They determined TDS ratios as the absolute values of $\Delta \mathrm{A}_{240 \mathrm{~nm}} / \Delta \mathrm{A}_{295 \mathrm{~nm}}, \Delta \mathrm{A}_{255 \mathrm{~nm}} / \Delta \mathrm{A}_{295 \mathrm{~nm}}$ and $\Delta \mathrm{A}_{275 \mathrm{~nm}} / \Delta \mathrm{A}_{295 \mathrm{~nm}}$, where $\Delta \mathrm{A}_{\lambda}$ is the difference between the absorbance above and below the melting point at a given $\lambda$, and it transpired that the values of the $\Delta \mathrm{A}_{240 \mathrm{~nm}} / \Delta \mathrm{A}_{295 \mathrm{~nm}}$ ratio for parallel quadruplexes exceeded 4 , and for anti-parallel folding it was below 2 .

Unfortunately, the shape of the TDS spectrum alone is not sufficient to prove quadruplex formation and it is always required to interpret this together with the data obtained from other methods such as CD or NMR spectroscopy. It is because the negative band which is characteristic of quadruplex structures can also be observed for Z-DNA, i-motif, Hoogsteen duplexes and pyrimidine triplex structures (Mergny et al., 2005b). However, the absence of this negative band provides evidence that the molecule does not fold into the quadruplex structure.

\section{Electrospray Ionization Mass Spectrometry (ESI-MS)}

Electrospray Ionization Mass Spectrometry (ESI-MS) was successfully employed for detection a G-quadruplex structure formation in 1993 (Goodlett et al., 1993). Since then the technique has been a useful tool in structural studies of G-quadruplexes, including strand stoichiometry (Joly et al., 2012; Borbone et al., 2011; Cui and Yuan, 2011; Rosu et al., 2002 and 2010; Zhou et al., 2007), quadruplex-ligand interactions (Rosu et al., 2008; Li et al., 2008; Gabelica et al., 2007), structural transitions (Ferreira et al., 2012; Amato et al., 2009 and 2011; Li et al., 2009), the kinetics of structure formation (Rosu et al., 2010; Gabelica et al., 2009) and many others (for a review, see Yuan et al., 2011).

Mass spectrometry relies on ionization of the compounds to generate charged molecules or molecule fragments in order to measure their mass-to-charge ratios $(\mathrm{m} / \mathrm{z})$. For structural studies of nucleic acids prevention of molecule fragmentation is often required. The electrospray ionization method allows structure destruction to be avoided during the measurement by keeping both the temperature of the capillary or Z-spray source and acceleration voltages in the transfer optics as low as possible. A very helpful experimental report containing sample preparation hints, preferred instrument settings 
A

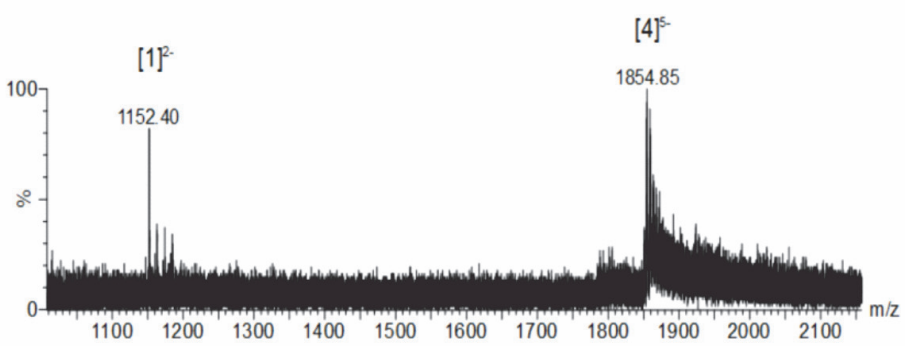

B

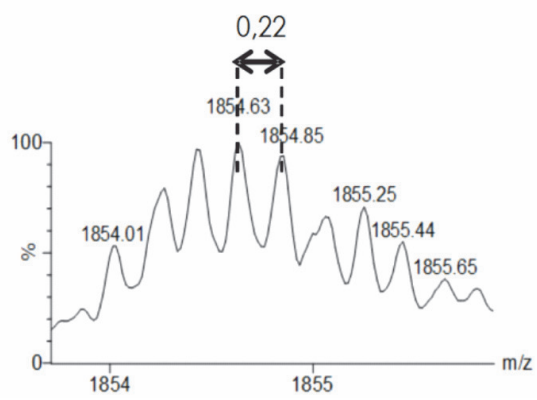

Fig. 6. Electrospray mass spectrum of $\mathrm{r}\left(\mathrm{AGGAGGA}_{4}\right.$ quadruplex (A) and the distance between isotopic peaks (B)

and recording procedures was prepared by V. Gabelica (Gabelica, 2010).

ESI-MS appears to be the method of choice for determination of the number of nucleic acid strands involved in a G-quadruplex formation and the number of ions adducted between its G-tetrad layers. Importantly, the negative ion mode is preferably used for recording the ESI-MS spectra of quadruplexes, because nucleic acid backbones are negatively charged in solution (fully deprotonated) (Gabelica, 2010).

In order to determine the number of nucleic acid strands involved in the formation of a particular structure it is necessary to determine the molecular weight (MW) of the ionized quadruplex, based on the measured $\mathrm{m} / z$ value. This value may also be used to calculate the number of cations located inside the G-quadruplex structure. Next, the information on the exact number of ions that are trapped inside the structure helps to predict the number of G-tetrad planes existing in the G-quadruplex structure. Sodium and potassium ions should be avoided in ESI-MS structural studies of G-quadruplexes. Although their presence between adjacent G-tetrads stabilizes the structure, they also tend to condense around negatively charged nucleic acid backbones during solvent droplet evaporation. This results in the occurrence of additional [DNA/RNA $\left.+n \mathrm{Na}^{+} / \mathrm{K}^{+}\right]$adducts that are heterogeneous in mass, which often leads to the loss of sensitivity and mass accuracy. To overcome this problem, $\mathrm{NH}_{4}^{+}$ions are usually used, in a typical concentration of $150 \mathrm{mM}$ and with $\mathrm{CH}_{3} \mathrm{COO}^{-}$as the counter-ions. Ammonium ions allow salt adducts to be avoided because complete solvent evaporation is followed by proton transfer between $\mathrm{NH}_{4}^{+}$and $\mathrm{PO}^{-}$from the negatively charged phosphodiester backbone, and in consequence ions that are not coordinated between G-tetrads evaporate as $\mathrm{NH}_{3}$ (Gabelica, 2010; Rosu et al., 2008). Usually the detected number of ammonium ions is one less than the number of G-tetrad planes involved in the structure formation, as only one ion is typically found between each pair of adjacent G-tetrads. This rule was clearly shown by F. Rosu et al., 2008. Determination of the number of adjacent G-tetrads may help in the understanding of the topology of the analyzed G-quadruplex structure.

To calculate the structural parameters the equation: $m / z=\left(n \times m_{\mathrm{NA}}+(t-1) \times 17-z\right) / z$ can be used $(n-$ the number of strands, $m_{\mathrm{NA}}$ - the molecular weight of neutral nucleic acid in Daltons, $t$ - the number of consecutive G-tetrads, $z$ - the charge of the ion). If the resolution of the mass spectra is sufficient and the isotopic distribution can be resolved, the charge can be easily calculated based on the rule that the distance between isotopic peaks is $1 / z$ (Rosu et al., 2008). For example, if the molecular weight of the $\mathrm{r}(\mathrm{AGGAGGA})$ molecule is $2306.50 \mathrm{Da}$, the $\mathrm{m} / z$ value is 1854.85 (Fig. $6 \mathrm{~A}$ ), and the distance between isotopic peaks is 0.2 (Fig. $6 \mathrm{~B}$ ), then the charge is 5 , the number of nucleic acid strands is 4 $(4 \times 2306.50=9226)$ and the number of ammonium ions sited between G-tetrads is equal to $3(1854.85 \times 5$ - 9226)/17 = 2.8). So, the analyzed quadruplex structure contains four adjacent G-tetrads.

ESI-MS spectra recorded for the samples prepared in aqueous solutions may exhibit quite low signal intensities. This happens mainly because of the high surface tension of the formed droplets, which slows down the evaporation processes and allows fewer ions to reach the detector. To obtain a higher signal intensity, methanol with a $10-20 \%(\mathrm{v} / \mathrm{v})$ final concentration is added to the sample 
immediately before injection into the mass spectrometer. Unfortunately, in some cases methanol was observed to influence the structure formation kinetics (Rosu et al., 2010). To be sure that the obtained results are reliable it is advisable to compare them with the spectra recorded for molecules in $100 \%$ aqueous solutions (Ferreira et al., 2012).

\section{Nuclear Magnetic Resonance Spectroscopy (NMR)}

As already mentioned, high-resolution NMR spectroscopy and X-ray crystallography can both be used to determine G-quadruplex structure with atomic resolution. However, only NMR spectroscopy allows determination of the structure in solution, a natural condition for molecules found in living cells.

G-quadruplexes give characteristic NMR spectra (Webba da Silva, 2007). The resonances corresponding to imino protons of guanine residues involved in G-tetrad formation appear at characteristic chemical shifts around 10.5-12 ppm (Fig. 7A). Usually, the number of the imino proton resonances is correlated with the number of guanosine residues involved in G-tetrad formation. This spectral region is separated from imino regions of other forms of nucleic acids, such as duplexes, single-strands, or other secondary structures. Therefore, this region of the NMR spectra provides a convenient method for monitoring not only the formation of a G-quadruplex structure but also its ligand binding interactions (Phan et al., 2005).

Another characteristic feature of the NMR spectra of quadruplexes is the very slow exchange of guanine imino protons with deuterated solvents. For example, the exchange of the solvent from $\mathrm{H}_{2} \mathrm{O}$ to $\mathrm{D}_{2} \mathrm{O}$ causes an immediate loss of exchangeable imino- and amino proton signals for duplex forms, while in quadruplexes a complete replacement of the hydrogen bonded atoms with deuterium generally occurs after many hours, sometimes even after a few weeks or months (Fig. 7B). This observation is often used to prove G-quadruplex formation. G-quadruplex structure depends not only on the nucleotide sequence but also on sample preparation and solvent conditions. Such factors as sample annealing and quenching, nucleic acid concentration (Trajkovski et al., 2012), temperature (Phan et al., 2003), salt type and concentration (Phan, 2010), the type of the buffer used and the presence of a co-solvent (Vorlickova et al., 2007)

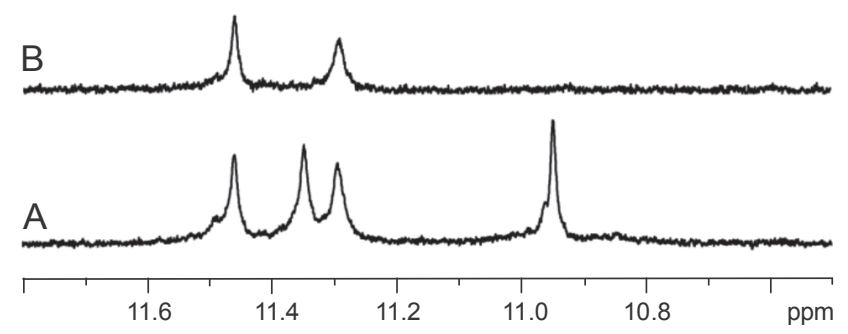

Fig. 7. Imino region of $\mathrm{r}(\mathrm{AGGAGGA})_{4}$ quadruplex in $90 \% \mathrm{H}_{2} \mathrm{O} / 10 \% \mathrm{D}_{2} \mathrm{O}(\mathrm{A})$ and after $12 \mathrm{~h}$ in $\mathrm{D}_{2} \mathrm{O}(\mathrm{B})$

can also affect the relative population and NMR spectra of G-quadruplexes.

In order to determine the three-dimensional structure of G-quadruplexes by NMR spectroscopy, the application of multidimensional methods is needed to allow the assignment of ${ }^{1} \mathrm{H},{ }^{13} \mathrm{C},{ }^{15} \mathrm{~N}$ and ${ }^{31} \mathrm{P}$ resonances. After the complete spectral assignment, the folding topology of a G-quadruplex is determined, followed by three-dimensional structure determination using NMR-derived distance restraints in combination with molecular dynamics calculations. However, successful structure determination by NMR spectroscopy is possible only for molecules which form a major stable structure with good NMR spectral properties in solution, i.e., sharp, well resolved lines. The coexistence of different forms in solution not only makes structural studies harder by significantly reducing the amount of available information, but usually makes it impossible to draw any conclusions. To improve the quality of NMR spectra and facilitate structural analysis, different approaches to obtain a single G-quadruplex conformation in solution have been established. It has been found that even minor changes of the oligonucleotide sequence at either the $5^{\prime}$ or $3^{\prime}$ ends may change the quality of the spectrum (Fig. 8) (Phan et al., 2007). In quadruplex studies a common practice is to screen many different oligonucleotide sequences until one is found which gives a good NMR spectrum and allows a structural analysis to be initiated (Phan et al., 2004, Ambrus et al., 2005, Phan et al., 2005). However, the introduction of such modification may result in a change of the quadruplex topology (Fig. 9). Alternatively, in order to force a single conformation in solution some guanosine residues can be substituted by 8 -bromoguanosine or inosine (Lim et al., 2009, Lim et al., 2010). In fact, even if the methods above are used, about $10 \%$ of higher-order structures as 


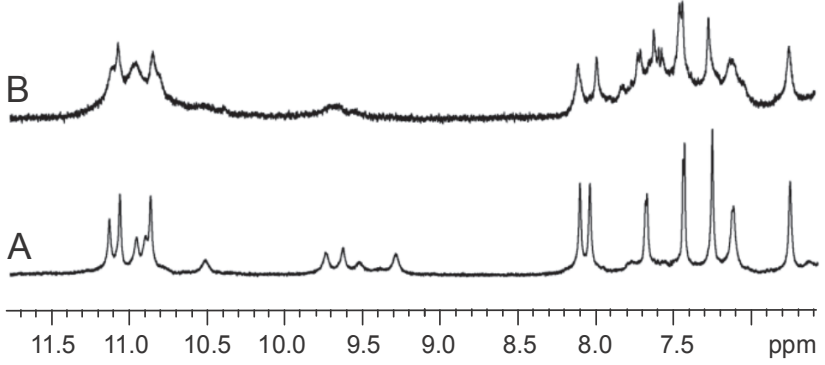

Fig. 8. The influence of the addition of $5^{\prime}$-phosphate group to $\mathrm{r}(\mathrm{UGGUGGU})_{4}$ quadruplex. The ${ }^{1} \mathrm{H}$ NMR spectrum of $r(\mathrm{UGGUGGU})_{4}(\mathrm{~A})$ and $\mathrm{r}(\mathrm{pUGGUGGU})_{4}(\mathrm{~B})$

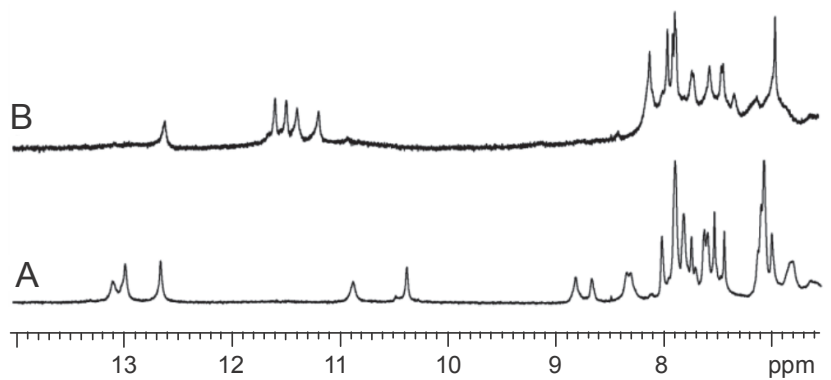

Fig. 9. The change of quadruplex topology after the substitution one guanosine residue by 8 -bromoguanosine, ${ }^{B r} \boldsymbol{G}$. The ${ }^{1} \mathrm{H}$ NMR

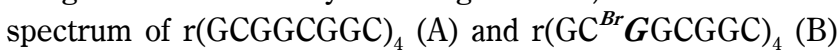

well as an alternative conformation background is usually observed at ${ }^{1} \mathrm{H}$ NMR spectra.

Two reviews on the application of NMR spectroscopy to study the structure, dynamics and interaction of G-quadruplexes have been recently published and many details concerning spectral assignment strategy, fold determination, structure calculation can be found there (Webba da Silva 2007, Adrian et al., 2012).

\section{Conclusions}

In addition to the methods presented above there is a wide range of other techniques that have been used to study G-quadruplex structures. These include biophysical methods such as FRET (Fluorescence Resonance Energy Transfer), DLS (Dynamic Light Scattering), DCS (Differential Scattering Calorimetry), biochemical techniques, such as gel electrophoresis or enzymatic probing and computational methods such as molecular dynamics. Nevertheless, in our opinion, the combined use of the methods described in this review have many advantages. UV and CD spectroscopies as well as mass spectrometry are convenient, fast, sensitive and informative experimental techniques, whereas NMR spectro- scopy provides the G-quadruplex structures with atomic resolution.

\section{Acknowledgments}

This work was supported by the Polish National Science Center (NCN) under Grant No. N N301 707040

\section{References}

Adrian M., Heddi B., Phan A.T., (2012) NMR spectroscopy of G-quadruplexes. Methods 57:11-24.

Alberti P., Bourdoncle A., Sacca B., Lacroix L., Mergny J.L. (2006) DNA nanomachines and nanostructures involving quadruplexes. Org. Biomol. Chem. 4: 3383-3391.

Amato J., Oliviero G., De Pauw E., Gabelica V. (2009) Hybridization of short complementary PNAs to G-quadruplex forming oligonucleotides: an electrospray mass spectrometry study. Biopolymers 91: 244-255

Amato J., Pagano B., Borbone N., Oliviero G., Gabelica V., De Pauw E., D’Errico S., Piccialli V., Varra M., Giancola C., Piccialli G., Mayol L. (2011) Targeting G-quadruplex structure in the human c-Kit promoter with short PNA sequences. Bioconjugate Chem. 22: 654-663

Ambrus A., Chen D., Dai J., Jones R.A., Yang D. (2005) Solution Structure of the Biologically Relevant G-Quadruplex Element in the Human c-MYC Promoter. Implications for G-Quadruplex Stabilization. Biochemistry 44: 2048-2058.

Avino A., Mazzini S., Ferreira R., Gargallo R., Marquez V.E., Eritja R. (2012) The effect on quadruplex tability of Northnucleoside derivatives in the loops of the thrombin-binding aptamer. Bioorg. Med. Hem. 20: 4186-4193.

Balasubramanian S., Neidle S. (2009) G-quadruplex nucleic acids as therapeutic targets. Curr. Opin. Chem. Biol. 13: 345-353.

Bang I. (1910) Untersuchungen uber die Guanylsaure, Biochem. Zeitschr. 26: 293-311.

Boczkowska M. (2002) Dichroizm kołowy kwasów nukleinowych Cz. I. Monomery. Wiad. Chem. 56: 39-56.

Borbone N., Amato J., Oliviero G., D’Atri V., Gabelica V., De Pauw E., Piccialli G., Mayol L. (2011) $d(C G G T G G T)$ forms an octameric parallel G-quadruplex via stacking of unusual $G(: C): G(: C): G(: C): G(: C)$ octads. Nucl. Acids Res. 39: 7848-7857.

Bugaut A., Balasubramanian S. (2012) 5'-UTR RNA G-quadruplexes: translation regulation and targeting. Nucl. Acids Res. 40: 4727-4741.

Burge S., Parkinson G. N., Hazel P., Todd A. K., Neidle S. (2006) Quadruplex DNA: sequence, topology and structure. Nucl. Acids Res. 34: 5402-5415.

Collie G.W., Parkinson G.N. (2011) The application of DNA and RNA G-quadruplexes to therapeutic medicines. Chem. Soc. Rev. 40: 5867-5892.

Cui X., Yuan G. (2011) Formation and recognition of G-quadruplex in promoter of c-myb oncogene by electrospray ionization mass spectrometry. J. Mass Spertrom. 46: 849855. 
Di Antonio M., Rodriguez R., Balasubramanian S. (2012) $E_{X^{-}}$ perimental approaches to identify cellular G-quadruplex structures and functions. Methods 57: 84-92.

Ferreira R., Marchand A., Gabelica V. (2012) Mass spectrometry and ion mobility spectrometry of G-quadruplexes. A study of solvent effects on dimer formation and structural transitions in the telomeric DNA sequence d(TAGGGTTAGGGT). Methods 57: 56-63.

Gabelica V., Baker E.S., Teulade-Fichou M.P., De Pauw E., Bowers M.T. (2007) Stabilization and structure of telomeric and c-myc region intramolecular $G$-quadruplex: The role of central cations and small planar ligands. J. Am. Soc. 129: 895-904.

Gabelica V., Rosu F., De Pauw E. (2009) A simple method to determine electrospray response factors of noncovalent complexes. Anal. Chem. 81: 6708-6715.

Gabelica V. (2010) Determination of equilibrium association constants of ligand-DNA complexes by electrospray mass spectrometry. Methods Mol. Biol. 613: 89-101.

Gatto B., Palumbo M., Sissi C. (2009) Nucleic Acid Aptamers Based on the G-Quadruplex Structure: Therapeutic and Diagnostic Potential. Curr. Med. Chem. 16: 1248-1265.

Gellert M., Lipsett M.N., Davies D.R. (1962) Helix formation by guanylic acid. Proc. Natl. Acad. Sci. USA 48: 20132018.

Goodlett D.R., Camp D.G., Hardin C.C., Corregan M., Smith R.D. (1993) Direct observation of a DNA quadruplex by electrospray ionization mass spectrometry. Biol. Mass Spectrom. 22: 181-183.

Gray D.M., Wen J.D., Gray C.W., Repges R., Repges C., Raabe G., Fleischhauer J. (2008) Measured and calculated CD spectra of G-quartets stacked with the same or opposite polarities. Chirality 20: 431-440.

Gros J., Rosu F., Amrane S., De Cian A., Gabelica V., Lacroix L., Mergny J.L. (2007) Guanines are quartet's best friend : impact of base substitutions on the kinetics and stability of tetramolecular quadruplexes. Nucl. Acids Res. 35: 3064-3075.

Guo L., Nie D., Qiu C., Zheng Q., Wu H., Ye P., Hao Y., Fu F., Chen G. (2012) A G-quadruplex based label-free fluorescent biosensor for lead ion. Biosens. Bioelectron. 35: 123127.

Huppert J.L., Balasubramanian S. (2005) Prevalence of quadruplexes in the human genome. Nuc. Acids Res. 33: 2908-2916.

Huppert J.L., Bugaut A., Kumari S., Balasubramanian S. (2008a) G-quadruplexes: the beginning and end of UTRS. Nuc. Acids Res. 36: 6260-6268.

Huppert J.L. (2008b) Four-stranded nucleic acids: structure, function and targeting of G-quadruplexes. Chem. Soc. Rev. 37: 1375-1384.

Joly L., Rosu F., Gabelica V. (2012) $d\left(T G_{n} T\right) D N A$ sequences do not necessarily form tetramolecular $G$ - quadruplexes. Chem. Commun., 48: 8386-8388.

Karsisiotis A.I., Hessari N.M., Novellino E., Spada G.P., Randazzo A., Webba da Silva M. (2011) Topological chara- cterization of nucleic acid G-quadruplexes by UV absorp tion and circular dichroism. Angew. Chem. Int. Ed. 50: 10645-10648.

Kypr J., Kejnovska I., Renciuk D., Vorlickova M. (2009) Circular dichroism and conformational polymorphism of DNA. Nuc. Acids Res. 37: 1713-1725.

Li H., Yuan G., Du D. (2008) Investigation of formation, recognition, stabilization, and conversion of dimeric $G$-quadruplexes of HIV-1 integrase inhibitors by electrospray ionization mass spectrometry. Eur. J. Mass Spectrom. 15: 731-737.

Li H., Liu Y.Q., Lin S., Yuan G. (2009) Spectroscopy probing of the formation, recognition and conversion of a $G$-quadruplex in the promoter region of the bcl-2 oncogene. Chem. Eur. J. 15: 2445-2452.

Lim K.W., Amrane S., Bouaziz S., Xu W., Mu Y., Patel D.J., Luu K.N., Phan A.T., (2009) Structure of the human telomere in $K^{+}$solution: a stable basket-type G-quadruplex with only two G-tetrad layers. J. Am. Chem. Soc. 131: 4301-4309.

Lim K.W., Lacroix L., Yue D.J., Lim J.K., Lim J.M., Phan A.T., (2010) Coex-istence of two distinct G-quadruplex conformations in the hTERT promoter. J. Am Chem. Soc. 132: 12331-12342.

Lipps H.J., Rhodes D. (2009) G-quadruplex structures: in vivo evidence and function, Tredns Cell Biol. 19: 414-422.

Liu W., Zhu H., Zheng B., Cheng S., Fu Y., Li W., Lau T.C., Liang H., (2012) Kinetics and mechanism of G-quadruplex formation and conformational switch in a G-quadruplex of PS2.M induced by $\mathrm{Pb}^{2+}$. Nucl. Acids Res. 40: 4229-4236.

Mergny J.L., Phan A.T., Lacroix L. (1998) Following G-quartet formation by $U V$ - spectroscopy. FEBS Lett. 435: 74-78.

Mergny J.L., De Cian A., Ghelab A., Sacca B. and Lacroix L. (2005a) Kinetics of tetramolecular quadruplexes. Nucl. Acids Res. 33: 81-94.

Mergny J.L., Li J., Lacroix L., Amrane S. and Chaires J.B. (2005b) Thermal difference spectra: a specific signature for nucleic acid structures. Nucl. Acids Res. 33: e138.

Mergny J.L., Lacroix L. (2009) UV melting of G-quadruplexes. Curr. Protoc. Nucl. Acids Chem. 17.1.1-17.1.15.

Miyoshi D., Sugimoto N. (2011) G-Quartet, G-Quadruplex, and G-Wire Regulated by Chemical Stimuli. In: DNA Nanotechnology: Methods and Protocols, eds. Zuccheri G., Samori B., Totowa: Humana Press Inc., New Jersey, p. 93-104.

Paramasivan S., Rujan I., Bolton P.H., (2007) Circular dichroism of quadruplex DNAs: applications to structure, cation effects and ligand binding. Methods 43: 324-331.

Pasternak A., Hernandez F.J., Rasmussen L.M., Vester B., Wengel J. (2011) Improved thrombin binding aptamer by incorporation of a single unlocked nucleic acid monomer. Nucl. Acids Res. 39: 1155-1164.

Phan A.T., Patel D.J. (2003). Two-repeat human telomeric d(TAGGGTTAGGGT) sequence forms interconverting parallel and antiparallel G-quadruplexes in solution: Distinct topologies, thermodynamic properties and folding/ unfolding kinetics. J. Am. Chem. Soc., 125: 15021-15027. 
Phan A.T., Modi Y.S., Patel D.J. (2004) Propeller-Type Parallel-Stranded G-Quadruplexes in the Human c-myc Promoter. J. Am. Chem. Soc. 126: 8710-8716.

Phan A.T., Kuryavyi V., Gaw H.Y., Patel D.J., (2005) Smallmolecule interaction with a five-guanine-tract $G$-quadruplex structure from the human MYC promoter. Nat. Chem. Biol. 1: 167-173.

Phan A.T., Kuryavyi V., Luu K.N., Patel J.D., (2007) Structure of two intramolecular G-quadruplexes formed by natural human telomere sequences in $K^{+}$solution. Nuc. Acids Res. 35: 6517-6525.

Phan A.T., (2010) Human telomeric G-quadruplex: structures of DNA and RNA sequences. FEBS J. 277: 1107-1117.

Rachwal P.A., Fox K.R. (2007) Quadruplex melting. Methods 43: 291-301.

Randazzo A., Spada G.P., Silva M.W. (2012) Circular Dichroism of Quadruplex Structures. Top. Curr. Chem. DOI: 10.1007/128_2012_331.

Rosu F., Gabelica V., Houssier C., Colson P., De Pauw E. (2002) Triplex and quadruplex DNA structures studied by electrospray mass spectrometry. Rapid Commun. Mass Spectrom. 16: 1729-1736.

Rosu F., De Pauw E., Gabelica V. (2008) Electrospray mass spectrometry to study drug-mucleic acids interactions. Biochimie 90: 1074 - 1087

Rosu F., Gabelica V., Poncelet H., De Pauw E. (2010) Tetramolecular G-quadruplex formation pathways studied by electrospray mass spectrometry. Nucl. Acids Res. 38: $5217-5225$

Sen D., Gilbert W. (1988) Formation of parallel four-stranded complexes by guanine- rich motifs in DNA and its implications for meiosis. Nature 334: 364-366.

Smargiasso N., Rosu F., Hsia W., Colson P., Baker E.S., Bowers M.T., De Pauw E., Gabelica V. (2008) G-quadruplex
DNA assemblies: loop length, cation identity, and multimer formation. J. Am. Chem. Soc. 130: 10208-10216.

Todd A.K., Johnston M., Neidle S. (2005) Highly prevalent putative quadruplex sequence motifs in human DNA. Nucl. Acids Res. 33: 2901-2907.

Trajkovski M., Webba da Silva M., Plavec J., (2012) Unique Structural Features of Interconverting Monomeric and Dimeric G-Quadruplexes Adopted by a Sequence from the Intron of the N-myc Gene. J. Am. Chem. Soc. 134: 41324141.

Vorlickova M., Bednarova K., Kejnovska I., Kypr J. (2007) Intramolecular and intermolecular guanine quadruplexes of DNA in aqueous salt and ethanol solutions. Biopolymers 86: $1-10$

Vorlickova M., Kejnovska I., Sagi J., Renciuk D., Bednarova K., Motlova J., Kypr J., (2012) Circular dichroism and guanine quadruplexes. Methods 57: 64-75.

Webba da Silva M., (2007) NMR methods for studying quadruplex nucleic acids. Methods 43: 264-277.

Wei C., Jia G., Zhou J., Han G., Li C. (2009) Evidence for the binding mode of porphyrins to G-quadruplex DNA. Phys. Chem. Chem. Phys. 11: 4025-4032.

Williamson J.R., Raghuraman M.K., Cech T.R. (1989) Monovalent cation-induced structure of telomeric DNA: The G-quartet model. Cell 59: 871-880.

Yuan G., Zhang Q., Zhou J., Li H. (2011) Mass spectrometry of G-quadruplex DNA: formation, recognition, property, conversion and conformation. Mass Spectrom. Rev. 30: 1121-1142.

Zhou J., Yuan G., Liu J.J., Zhan C.G. (2007) Formation and stability of G-quadruplexex self-assembled from guanine rich strands. Nucl. Acids Res. 32: 1-9. 